\title{
A Modified Fused Floating Point Three Term Adder
}

\section{Neeraja P K, Ramadass Narayanadass}

\begin{abstract}
This paper is about a modified architecture for a fused floating point three term adder. The important feature of a fused floating-point three-term adder is its ability to do multiple additions in same block to get better performance as well as accuracy compared to a conventional discrete floating point adder. The parallel prefix adder is one amongst the fastest adders and out of which the han-carlson adder represents a blend of the kogge-stone adders and brent-kung adder. In this work, han carlson adder is used to enhance the performance of the three term adder along with various optimization techniques. The adder is implemented using Verilog language in Xilinx ISE Design suite 14.2 and all Simulations are carried out in Isim simulator. Synthesis is done using Cadencetool.
\end{abstract}

Keywords-Floating point adder, Parallel prefix addition, Kogge Stone adder, Han Carlson adder.

\section{INTRODUCTION}

Among most of the DSP implementations, multiple floatingpoint additions are executed consecutively. Floating point calculates a way of expressing an estimation of real number in such a way that it can uphold wide scope of values. It is described in IEEE-754 Standard for the floating point arithmetic[1]. Mostly, the numbers are expressed approximately to a specific number of significant digits and scaled utilizing an exponent. The base can be 2, 6 or 10 . Generally, a typical number can be expressed as:

$(-1)$ sign $\mathrm{x}$ significant digits $\mathrm{x}$ base $\mathrm{exponent}$

Main feature of floating-point multi-term adder is that it can take different operands and can execute numerous additions with an operation to produce a sum. There are two methods to design the floating point three-term adder namely a) Discrete floating-point three-term adder and b) Fused floating-point three-term adder. Complex processes like alignment, normalization and rounding are essential for floating point operation, but this increases area, power consumption and latency. So as to eliminate such issues, fused floating-point units have been proposed, that perform numerous operations in a single block to reduce the area, power consumption and latency. Some problems for the implementation of the fused floating point three term adder are studied in the past work [3], [4]: 1) Complex exponent processing and significand alignment, 2)

Revised Manuscript Received on October 25, 2020

*CorrespondenceAuthor

Neeraja $\mathbf{P} \mathbf{K}$, Department of Electronics and Communication Engineering,CollegeofEngineeringGuindy,AnnaUniversity,Chennai,India. Email:neerajapkn@gmail.com

Ramadass Narayanadass*, Department of Electronics and Communication Engineering, College of Engineering Guindy, Anna University, Chennai, India. Email: ramadassn@annauniv.edu

(c) The Authors. Published by Blue Eyes Intelligence Engineering and Sciences Publication (BEIESP). This is an open access article under the CC BY-NC-ND license (http://creativecommons.org/licenses/by-nc-nd/4.0/)
Significand addition followed by complementation, 3) Large precision significand addition, 4) Massive cancellation management and 5) Complex round processing. Here in this work, such issues are addressed as well as performance is enhanced by introducing many optimization techniques and by modifying the type of parallel prefix adderused.
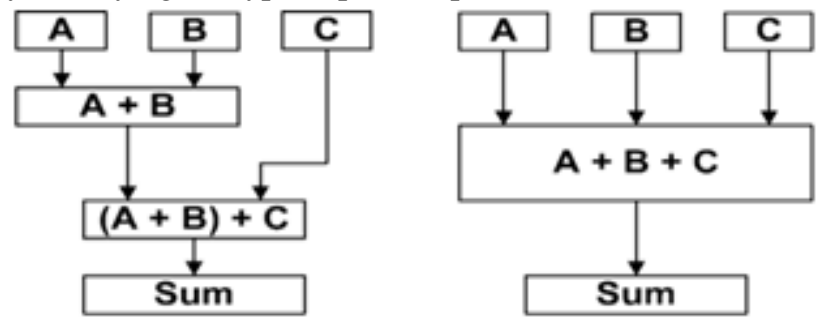

Fig. 1 Discrete Vs Fused floating point three term adders Section II explains the traditional fused floating-point three term adder. Improved architectures for the fused floating point three term adder design are presented in next section. Here, various optimization techniques are utilized to enhance the performance of the fused floating point three term adder and are briefly described in section III [2]. Based on a data flow analysis, modification is applied to the improved fused floating-point three-term adder In section IV, the proposed design is implemented for single precision. For this work han-carlson adder is used as a parallel prefix adder that is a combination of brent-kung adder and kogge stone adders section $\mathrm{V}$ gives results and conclusion is given in sectionVI.

\section{CONVENTIONAL FUSED FLOATINGPOINT THREE TERMADDERS}

In order to implement a floating point three term adder, it is possible to fuse two identical floating point adders. One among the high performance floating point adders [8]-[10] can be used for the discrete design. The discrete floating point adder takes much more area compared to single term adder even though it has the capability to do multi term additions. For decreasing the overhead, fused floating point three term adders are introduced, that can carry out two additions in a single block [3], [4]. In order to decrease the area, power consumption and delay, a common logic is shared by the adder . Fig. 1 shows comparison of the discrete design and presented fused design. Moreover, the fused floating point three termadder improves the accuracy of design. The discrete floating point three term adderperforms rounding twice whereas the fused floating point three term adder do the rounding only once, thereby improving the accuracy.

Published By: and Sciences Publication

(C) Copyright: All rights reserved.

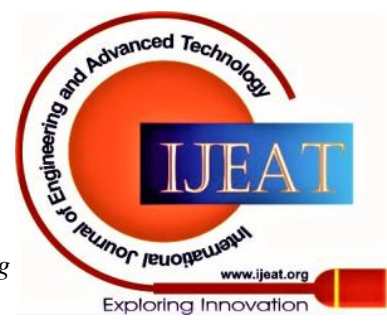




\section{A Modified Fused Floating Point Three Term Adder}

Fig. 2 shows a typical fused floating point three term adder [3], [4]. This adder takes three operands and performs the two additions at once.

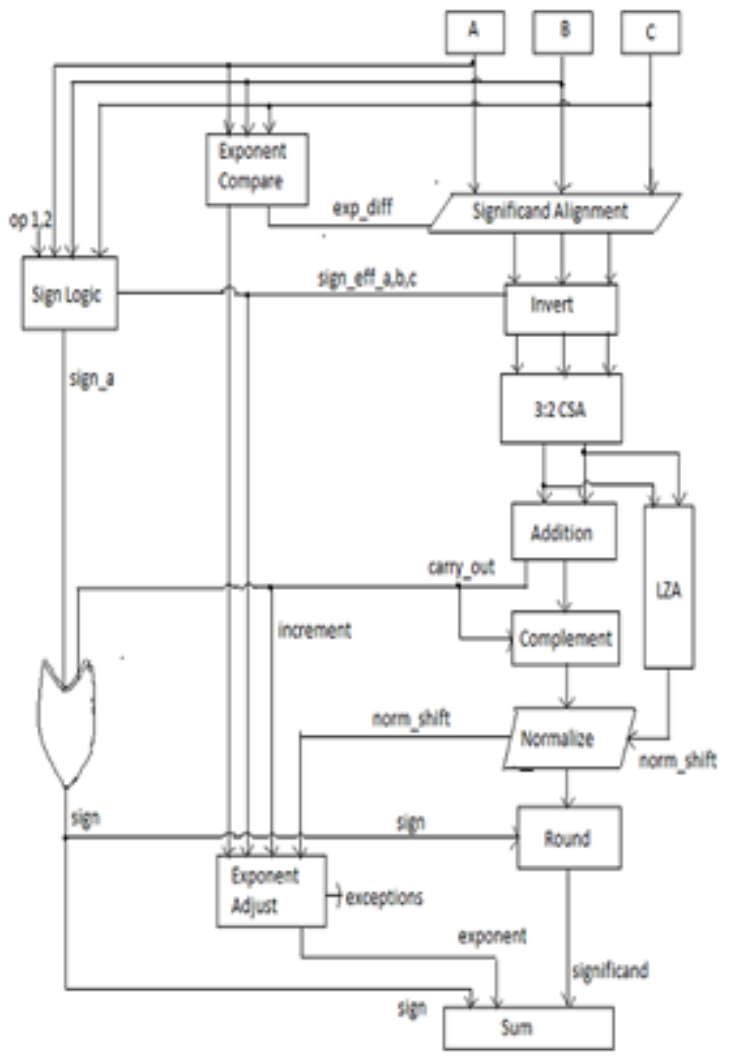

Fig. 2 Typical fused floating point three-term adder

\section{OPTIMIZATIONTECHNIQUES}

The conventional fused floating point three term adder lessens the latency, area and power consumption compared to the discrete version. In this section, a few optimizations areexplainedthatcanbeusedforimprovingtheperformance of the adder design: 1) A new exponent compare and significand alignment method 2) Dual reduction 3) Early normalization 4) Three input LZA and 5) Compound addition androunding.

1) Novel exponent compare with significand alignment method is presented. Here, three pairs of exponent differences are assessed in parallel and differences are utilized for significand alignment. The exponent difference evaluation and also significand alignment can be overlapped by shifting the significands using partial difference results. The control logic is responsible for finding out the largest exponent and three aligned significands. At the same time, exponent processing and significand alignment are performed. This thereby can lessen thelatency.

2) Two significand pairs such as positive as well as negative significand can be generated by two reduction trees. Positive significand pair is chosen based on the significand comparison. This method is called Dual reduction. So, complementation after the significand addition canbe skipped as the chosen significand pair produces a positive sum, which reduces the delay.

3) Early normalization is applied to decrease the significand addition size. Adder size is then reduced significantly by performing the normalization before the significand addition. Latency is again reduced

afterrounding.

As the normalization is done before significand addition, the Leading Zero Anticipation (LZA) as well as normalization are on the critical path. A three-input LZA is presented the $3: 2$ reductiontrees.

For fast rounding, compound addition is utilized.Rounded and unrounded sums are resulted by compound addition and the round logic finds out the accurate result so that the delay of the rounding ishidden.

\section{FUSED FLOATING POINT THREE TERM ADDER USING HAN CARLSONADDER}

In the conventional fused floating point three term adder, several optimization techniques have been used. The first part of significand addition is performed after the reduction to balance with the delay of LZA. The necessity for an adder is that it is fast as well as efficient according to power consumption and chip area. So here comes the advantage of parallel prefix adders. It is a technique for improving the speed of addition.

\section{A. Kogge Stoneadder:}

A Kogge stone adder has been utilized as a parallel prefix $r$ in the improved adder architecture. The Kogge Stone w logic depth, high node count, and less fan out. High nodecountimpliesabiggerareawhereasthelowlogicdepth and minimal fanout helps for faster performance. Mainly there are three computational stages in Kogge Stone Adder.

2. Carry generationnetwork

3. Postprocessing

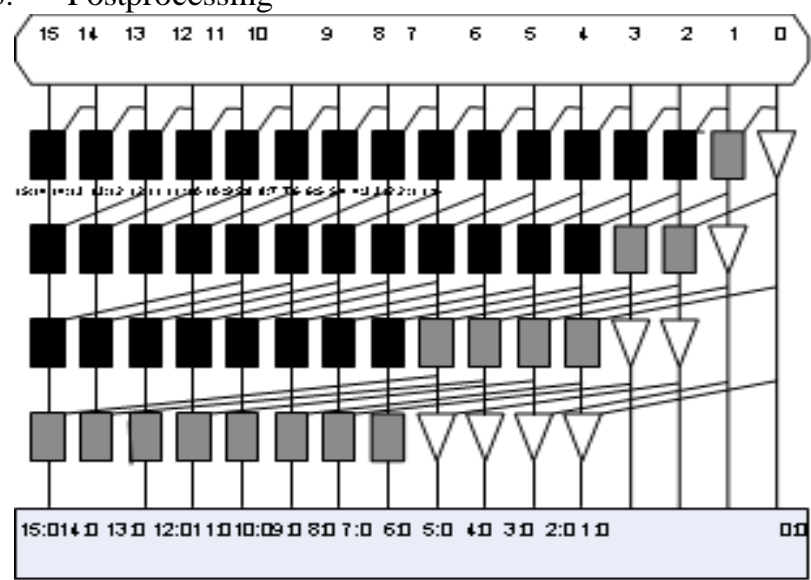

Fig. 316 bit Kogge Stone adder

$$
\begin{aligned}
& \mathrm{P}_{\mathrm{i}}=\mathrm{a}_{\mathrm{i}} \bigoplus_{\mathrm{b}_{\mathrm{i}}} \\
& \mathrm{g}_{\mathrm{i}}=\mathrm{a}_{\mathrm{i}} \mathrm{b}_{\mathrm{i}} \\
& P_{1}^{\mathrm{j}}=p_{i} p_{i-1} \ldots p_{i+\left(2^{j}-1\right)} \\
& \left.\mathrm{G}_{\mathrm{i}}^{\mathrm{j}}=\mathrm{g}_{\mathrm{i}}+\mathrm{g}_{\mathrm{i}-1} \mathrm{p}_{\mathrm{i}}+\ldots+\mathrm{g}_{\mathrm{i}-(2 \mathrm{j}-1)}^{\mathrm{j}}\right) \mathrm{p}_{\mathrm{i}} \mathrm{p}_{\mathrm{i}-1} \ldots \mathrm{p}_{\mathrm{i}-\left(2 \mathrm{2}_{-2}\right)}^{\mathrm{j}}
\end{aligned}
$$


Kogge Stone construction that takes log2n stages and the Brent kung construction that takes $2 \log _{2} \mathrm{n}-1$ stages[5,6]. Main advantage of Brunt kung design is that it takes a lesser area. whereaiandbiarei ${ }^{\text {th }}$ bitsoftwosignificandsandjisthe level of the prefix tree adder. But still Kogge Stone adder faces issues with its complex wiring circuitry. In the modified fusedfloating point three term adder, a Han Carlson adder is being used. Calculation of Carry generate as well as Propagatesignals Calculation of all Carry signals inparallel Calculation of last sum as well as carry signals

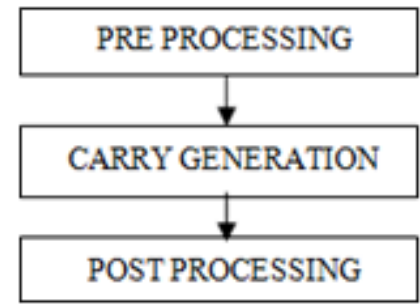

Fig. 4 Parallel Prefix mechanism

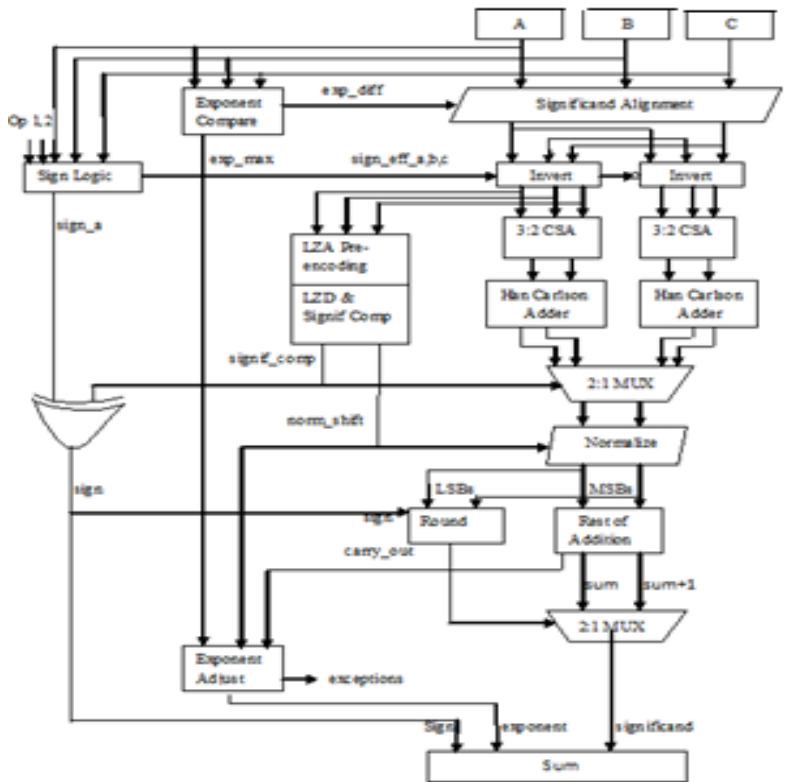

Fig. 5 A modified fused floating point three term adder

B. Han Carlsonadder:

Han Carlson adders are the group of networks comes in middle of Kogge stone and Brent Kung adders. Here, for 54 bit addition, Han Carlson adder is utilized. This sort of computation gives a speed nearer to Kogge Stone adder. A hybridversionofparallelprefix addersisputforwardedby $\mathrm{T}$. Han and D.A. Carlson by utilizing two designs like

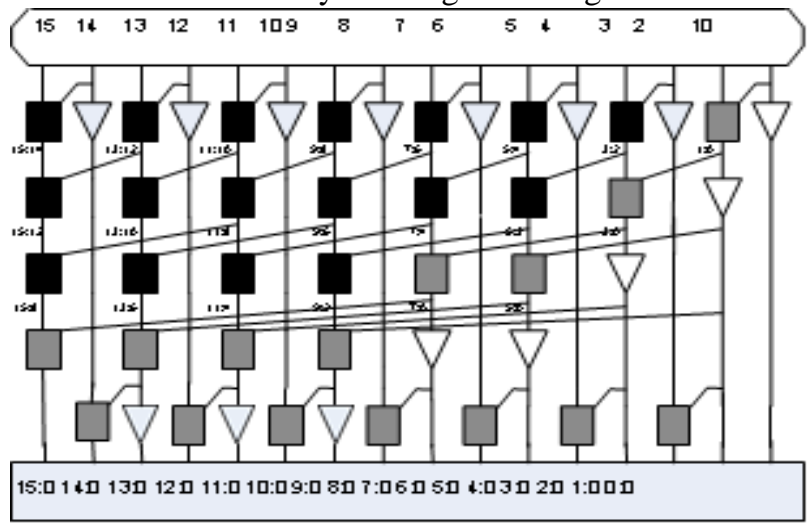

Fig. 616 bit Han Carlson adder

Thus Han Carlson adder has best features; high speed

Retrieval Number: 100.1/ijeat.A19081010120
obtainedfromKoggestoneadderandlowareobtainedfrom Brunt Kung design. Both features are combined to provide a a better speed with low complexity. This 16 bit han Carlson adder has five stages out of which three stages in the middle is similar to the kogge stone structure. Number of cells used by this adder is less compared to that used by kogge stone adder. And also this adder has shorter span wires so that wiring complexity is lesser than that inkogge stone structure [7]. The pseudo code for Han Carlson adder can be easily obtained by modifying that of Kogge Stone adder. The number of carry stages is log $2 \mathrm{n}+1$ where as for KoggeStone adder, it is log2n.Fig.7 shows method of Computation of carry in different stages.

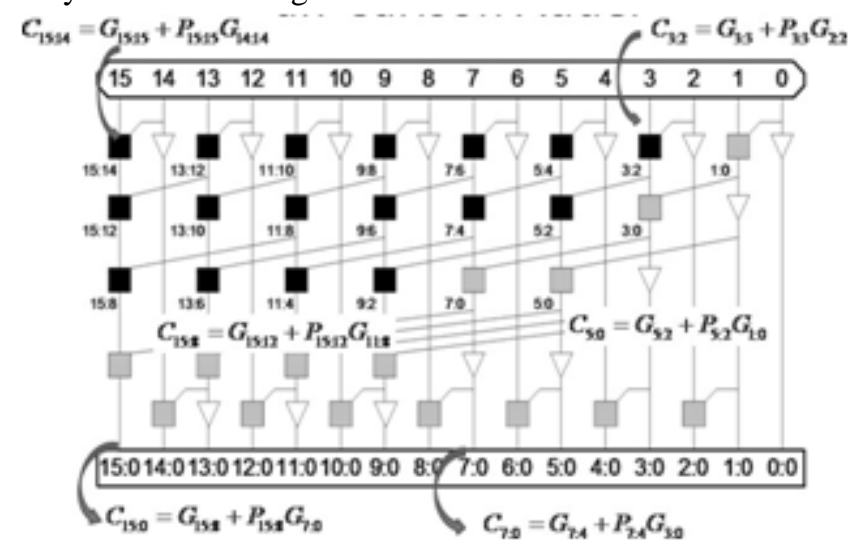

Fig.7 Carry Computation method of Han Carlson Adder

\section{RESULTS}

The proposed fused floating point three term adder is designed using

Verilog language in Xilinx ISE

Design suite 14.2.

ISim simulator is utilized for performing simulations. Later, the performance of conventional and proposed adders are analyzed and compared. Implementation code for 54-bit Kogge Stone adder as well as Han Carlson adder are developed in this work and the respective values of delayand area are measured. Table-I shows the algorithm analysis of parallelprefixaddersandTable-IIshowsthesynthesisresult.

Result comparisons are shown in tables 3,4. Simulation waveform results are shown in figures 7,8 and9.

Table-I: Algorithm Analysis of PPA's

\begin{tabular}{|c|l|l|c|c|}
\hline $\begin{array}{l}\text { Type of } \\
\text { PPA }\end{array}$ & $\begin{array}{l}\text { Logic } \\
\text { level }\end{array}$ & Area & $\begin{array}{c}\text { Fan } \\
\text { Out }\end{array}$ & $\begin{array}{c}\text { Wire } \\
\text { track }\end{array}$ \\
\hline $\begin{array}{c}\text { Kogge } \\
\text { Stone }\end{array}$ & $\log _{2} \mathrm{n}$ & $\mathrm{n} \log _{2} \mathrm{n}-\mathrm{n}+1$ & 2 & $\mathrm{n} / 2$ \\
\hline $\begin{array}{c}\text { Han } \\
\text { Carlson }\end{array}$ & $\log _{2} \mathrm{n}$ & $(\mathrm{n} / 2) \log _{2} \mathrm{n}$ & 2 & $\mathrm{n} / 4$ \\
\hline
\end{tabular}

Simulation Results:

Published By:

Blue Eyes Intelligence Engineering and Sciences Publication

(C) Copyright: All rights reserved.

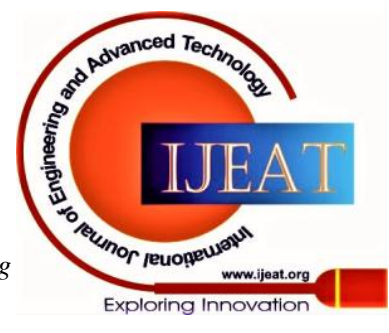




\section{A Modified Fused Floating Point Three Term Adder}

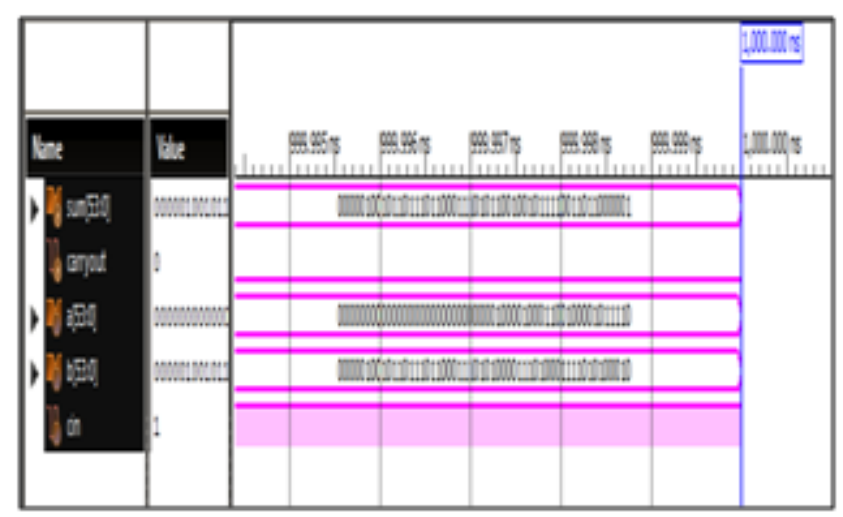

Fig.7 KSA Simulation output

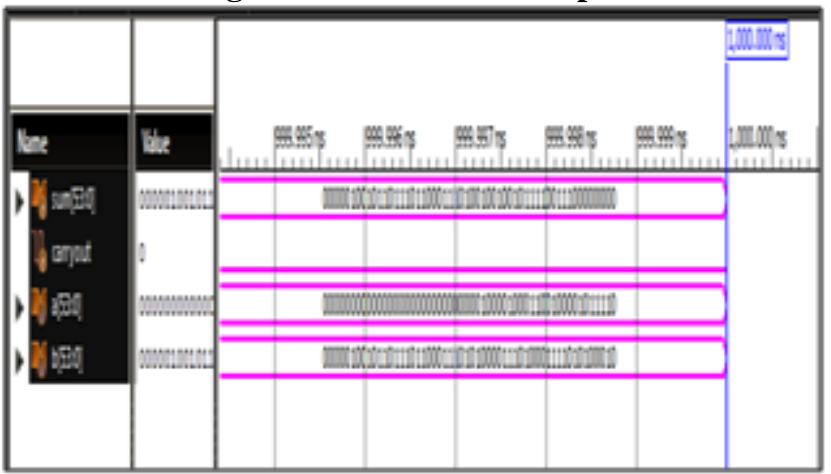

Fig.8 Han Carlson adder Simulation output Table-II: Synthesis Result

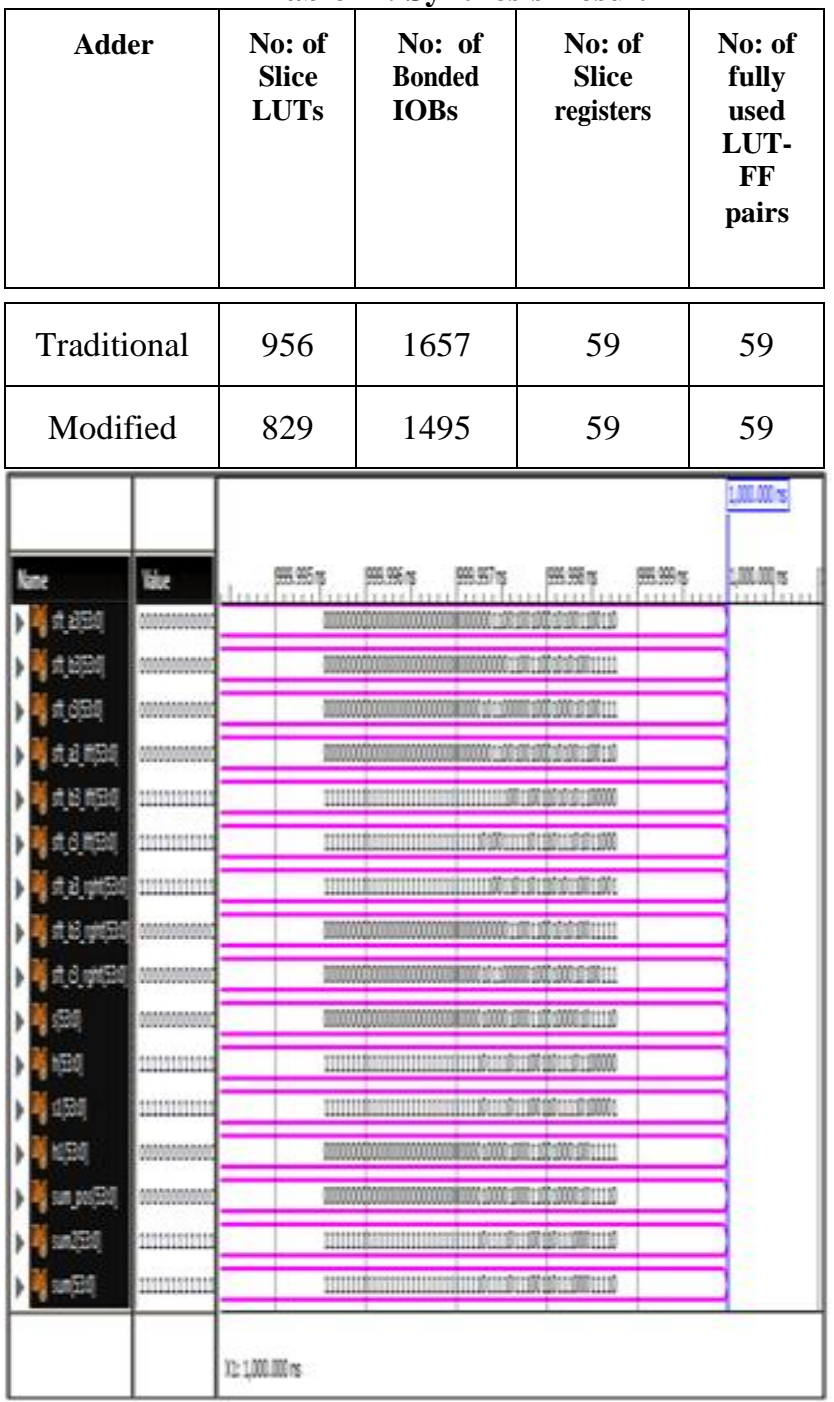

Fig.9 Simulation result of proposed adder
Both Kogge stone andHan Carlson adders require parallel wiring for wide bit adders. Han Carlson is so good as it requires only half number of columns when the interconnect is considered. Kogge stone tree has least number of logic levels; yet difficult to propagate as well as generate whereas Han Carlson has more number oflogic levels; yet less number ofcells.

Table-III: Result Comparison of PPAs

\begin{tabular}{|c|c|c|c|}
\hline $\begin{array}{c}\text { Parallel Prefix } \\
\text { Adder Type }\end{array}$ & Cell Area & $\begin{array}{c}\text { Leakage } \\
\text { Power } \\
\text { (nW) }\end{array}$ & Delay \\
\hline Kogge Stone & 1280 & 316 & $+0528 \mathrm{~F}$ \\
\hline Han Carlson & 1071 & 182 & $\begin{array}{c}+0 \\
6168 \mathrm{~F}\end{array}$ \\
\hline
\end{tabular}

Cell area and Leakage power consumption are more in Kogge stoneadder compared to Han Carlson adder.

Table-IV: Comparison of Traditional and Modified adder results

\begin{tabular}{|c|c|c|c|}
\hline Adder & $\begin{array}{c}\text { Cell } \\
\text { Area }\end{array}$ & $\begin{array}{c}\text { Leakage } \\
\text { Power } \\
\text { (nW) }\end{array}$ & $\begin{array}{c}\text { Combinational } \\
\text { Path Delay(ns) }\end{array}$ \\
\hline Traditional & 6973 & 1415 & 3.298 \\
\hline Modified & 5780 & 930 & 3.311 \\
\hline
\end{tabular}

\section{FUTUREWORK}

The work carried out in this paper can be extended to perform addition with terms more than three operands. Pipelining can also be employed along with other algorithms and optimization techniques discussed in the paper. Physical design implementation of the resulting adder also comes in the scope of future work.

\section{CONCLUSION}

In this paper, a modified fused floating point three term adder is designed using a parallel prefix adder calledHan Carlson adder. Various critical issues were there for the conventional three term adder. Those issues have been resolved by modified fused floating point three term adder. Han Carlson adder that is having less wiring complexity than Kogge Stone adder is used. The improved architecture reduces number of cells, area, and leakage power. But there a slight increase in delay is observed.

\section{REFERENCES}

1. IEEE Standard for Floating-Point Arithmetic ANSI/IEEE Standard 754-2008, IEEE, Inc.,2008.

2. J.SohnandE.E.Swartzlander,Jr.,"'AFusedFloating-PointThree- Term Adder", IEEE Transactions On Circuits And Systems-I: Regular Papers, Vol. 61, No. 10, October2014.

3. A. Tenca, "Multi-operand floating-point addition," in Proc. 21st Symp. Computer Arithmetic, 2009, pp.161-168.

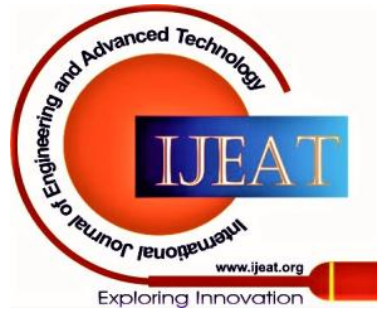


4. Y. Tao, G. Deyuan, F. Xiaoya, and R. Xianglong, "Three-operand floating-point adder," in Pro. 12th IEEE Int. Conf. Comput. Inf. Technol., 2012, pp. 192-196.

5. Swapna K. Gedam and Pravin P. Zode, "Parallel Prefix Han-Carlson Adder", International Journal of Research in Engineering and AppliedSciences.

6. Sreenivaas Muthyala Sudhakar, Kumar P. Chidambaram andEarl

E. Swartzlander Jr. "Hybrid Han-Carlson Adder", 978-1-4673-2527$1 / 12 / \$ 31.00 \odot 2012$ IEEE

7. Geeta Rani, Sachin Kumar," Delay Analysis of Parallel-Prefix Adders", International Journal of Science and Research(IJSR)

8. M. P. Farmwald, "On the Design of High Performance Digital Arithmetic Units,"Ph.D. dissertation, Computer Science, Stanford University, Stanford, CA, USA, 1981.

9. S.F.Oberman, H.Al-Twaijry, andM.J. Flynn,"The SNA Pproject: Designoffloatingpointarithmeticunits,"inProc.14thIEEESymp. Computer Arithmetic, 1997, pp.156-165.

10. P. M. Seidel and G. Even, "Delay-optimized implementation of IEEE floating-point addition," IEEE Trans. Computers, vol. 53, no. 2, pp. 97-113, Feb. 2004.

\section{AUTHORS PROFILE}

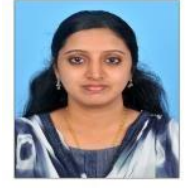

Neeraja P K was born in Kozhikode, Kerala, India in 1991. She received the B.Tech degree in Electronics and Communication Engineering from Kerala University in 2013 and M.E degree in VLSI design from Anna University, Chennai, India in 2016. She has completed Advanced diploma course in VLSI Physical Design from NIELIT, Calicut, India in 2019. She has worked as VLSI/Matlab Developer in Softroniics, Calicut and has completed training from Infosys, Mysore, Karnataka. She has also pursued an internship with Utkarshini Edutech in the field of Technical research- Digital Logic. Her research interests include VLSI design, Image processing and Signal Processing.

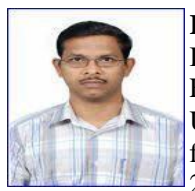

Ramadass Narayanadass was born in Chennai, Tamilnadu, India in 1975. He received the B.E degree in Electrical and Electronics Engineering from Madras Universityin1997,M.EdegreeinAppliedElectronicsand Ph.D from Anna University, Chennai, India in 2001 and 2008,respectively.HehasbeenassociatedwiththeFaculty

of Information and Communication Engineering, Anna University, Chennai, since2001.HeiscurrentlyanAssociateProfessorinDepartmentofElectronics and Communication Engineering at Anna University, Chennai.

His research interests include Embedded Systems, VLSI design and Reconfigurable Computing.

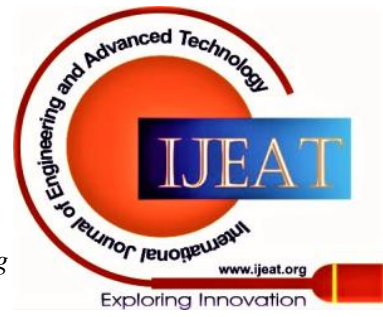

